\title{
On the manipulation of ferroelectric and ferroelastic domains at the nanoscale
}

Colm Durkan*, Jose Angel Garcia-Melendrez and Long Ding

Nanoscience Centre, University of Cambridge

$11 \mathrm{JJ}$ Thomson Avenue

Cambridge

CB3 0FF

*: corresponding author, email: cd229@eng.cam.ac.uk

\begin{abstract}
The distribution and evolution of ferroelectric and ferroelastic nanodomains in a polycrystalline (001)-oriented thin film of the simple multi-ferroic $\mathrm{PbZr}_{0.3} \mathrm{Ti}_{0.7} \mathrm{O}_{3}(\mathrm{PZT})$ is presented. With an external electric field applied between the probe tip and the PZT sample, ferroelastic domains were switched by rotating both in- and out-of-plane, with a partial conversion from $a$-oriented regions to $c$-oriented regions. After multiple such cycles, grains were observed to buckle as a direct consequence of the lateral size change arising from the conversion towards complete $c$-orientation. The factors determining the measured deflection of the cantilever in Piezoresponse force microscopy were explored, highlighting the conditions under which quantitative or qualitative information may be obtained.
\end{abstract}

Keywords: Ferroelectric, piezoelectric, domain, microscopy 


\section{Introduction}

Ferroelectric thin films have been extensively studied over the past several decades due to the wide application space in which they have been utilised, either for their ferroelectric or their piezoelectric properties [1-3]. With the increasing interest in the peculiar properties of domain walls and ferroelastic domains, both of which have characteristic lengthscales well below $10 \mathrm{~nm}$, it is becoming ever more important to have reliable tools to characterise and study these systems with near atomic resolution. A significant amount of information can be gleaned from electron microscopy, and with its ultimate resolution of a few picometres, it is possible to map the polarisation in ferroelectric materials at and below the unit-cell level [4-6]. However, it is not possible to probe the response of these materials to a local external influence such as pressure or an electric field, or to look at "real" samples - it is limited to studying free-standing films a few nm thick. With resonant-mode (i.e. operating at or near the in-contact resonant frequency of the AFM cantilever) Piezoresponse Force Microscopy (PFM), sub-nanometre resolution images of both ferroelastic and ferroelectric domains and their respective walls can be obtained alongside the topography, and the spatial distribution of the domains can be manipulated using the precise positioning capability of the electric probe tip. This resolution is due to the increased signalto-noise ratio relative to conventional (non-resonant) PFM. The amplitude and phase of the cantilever oscillation are detected and recorded as magnitude and phase images, respectively, from which we can extract information regarding the distribution of polarization within the material, at least within the vicinity of the surface.

Due to its high dielectric constant and unrivalled electromechanical coupling, Lead zirconate titanate (PZT) is widely used in both ferroelectric random access memory (RAM) devices, as well as surface acoustic wave filters, actuators and a range of sensors. It is also 
relatively straightforward to explore by PFM, which can be seen by looking at the rise in the number of papers on the topic that have been published over the past 15 years.

Driven by the desire to follow Moore's law of miniaturization in modern technology and to develop more-than-Moore devices, understanding ferroelectric materials at the nanoscale is very much in demand, as to do so will help to enable the production of smaller, faster, less power-hungry components. In polycrystalline ferroelectric PZT thin films, the tetragonal unit cells can form periodic stripes of $a$-domains and $c$-domains, known as polytwins, or as ferroelastic domains. This happens in the presence of mechanical stress, which arises due to (i) lattice mismatch between the PZT and the substrate and (ii) substrate clamping - the bottom surface of the film is constrained and cannot deform piezoelectrically, so in the presence of an applied electric field, the film becomes highly strained. This strain gets released by the material spontaneously forming periodic planar defects starting at the base, and spanning the thickness of the film, with a periodicity of anywhere from a few $\mathrm{nm}$ to several microns. Recently, it has been shown that certain ferroelectric and ferroelastic domain walls can exhibit electrical conductivity, depending on their topological parameters [7-9]. The long-held assumption that neighbouring unit cells align head-to-tail has also come into question. It is only with the advent of advanced imaging and measurement tools at the nanoscale that it has become possible to explore the new physics displayed by these correlated systems. Hence a further understanding of ferroelectric materials, especially topological defects and the ability to controllably affect nanodomain engineering, is crucial.

The tool used here to probe and manipulate the polarization, PFM, has been used for over 15 years and is now a mature technique in the nanomechanics toolbox, capable of high resolution and quantitative measurement [10-21]. 


\section{Experimental methods}

We chose a sol-gel grown polycrystalline $\mathrm{PbZr}_{0.3} \mathrm{Ti}_{0.7} \mathrm{O}_{3}(\mathrm{PZT})$ thin film for its high dielectric constant ( 1000) and large electromechanical coupling (in theory this can be up to $50 \%$ ).

The particular film used here was (001) oriented and $190 \mathrm{~nm}$ thick, on an Ir back electrode, as reported in reference [11]. This phase is tetragonal at room temperature with unit-cell dimensions along the $a$ and $c$ directions being $0.4036 \mathrm{~nm}$ and $0.4146 \mathrm{~nm}$, respectively. The AFM used in these experiments was a Solver Pro-M from NT-MDT, and the tips used were ElectriCont-G from BudgetSensors, with a nominal stiffness of $0.2 \mathrm{~N} / \mathrm{m}$, and a conductive $\mathrm{Cr} / \mathrm{Pt}$ coating on both sides of the cantilever.

\section{Experimental. I - Semi-quantitative PFM on static domains}

In PFM, a bias voltage, $V_{t i p}=V_{d c}+V_{a c} \sin \omega t$, is applied between the conducting AFM tip and the bottom electrode upon which the piezoelectric film is deposited, as depicted in Fig. 1 , where $V_{d c}$ is the dc potential difference between the tip and sample. This has two components: the contact potential difference arising from differences in the work function of tip and sample, and any voltage bias deliberately applied to the tip or sample. The amplitude and phase of the resulting cantilever oscillation at the drive frequency $\omega$ are measured. A detailed analysis of the relevant image formation mechanisms has already been developed and presented by several authors [22-24], taking into account the field distribution, the tipsample junction geometry and the indentation of the sample by the tip. The voltage $V_{\text {tip }}$ creates an electric field, $E_{t o t}$, where $E_{t o t}=E_{d c}+E_{a c} \sin \omega t$, which has four effects, namely (1) electrostriction, (2) local (at the tip apex) and non-local (over the body of the cantilever) Coulomb interactions, (3) piezoelectric deformation of the surface and (4) cantilever buckling due to in-plane motion of the tip induced by piezoelectric shearing of the sample. We will 
consider the relative effect of these terms later within the context of our experimental results, to aid image interpretation.

In the ferroelectric-ferroelastic biferroic material we are exploring, the $180^{\circ}, c-$ oriented ferroelectric domains appear as bright and dark areas in PFM phase images. The non- $180^{\circ}, a$-oriented (ferroelastic) domains are observed in magnitude images as bundles of polytwins at specific, well-defined orientations, that exhibit order over distances of up to several microns, often spanning several grains. Ferroelectric domains generally do not show up in magnitude images as they produce the same cantilever deflection irrespective of orientation (for the case of (001)-oriented films) apart from at the domain walls. Given the symmetry of the electric field from the tip, when the tip is on top of a $c^{+} / c^{-}$domain wall, the regions of the sample on either side of the wall will be moving in opposite directions, and the Coulomb force will also be opposite for each region, so at the very centre, all contributions apart from electrostriction will cancel out and the net cantilever motion will be zero. Both ferroelectric and ferroelastic domains can be electrically switched using the AFM tip, although the mechanisms and timescales behind both are different [25]. Ferroelectric domains are formed by aligning the polarisation of groups of unit cells towards a given direction to compensate the electrostatic contribution to the free energy; ferroelastic domains are, on the other hand, formed to release the mechanical stress in each grain caused by (i) lattice mismatch of the PZT and the substrate, (ii) defects within the material and (iii) lateral forces between adjacent grains which push against each other whilst undergoing piezoelectric and electrostrictive deformations. Superimposed on this is the flexoelectric effect whereby strain gradients give rise to an additional polarisation (in the direction of the gradient), which is observed locally as a rotation of the polarisation away from the crystallographically expected orientations [26]. 
Figure 2 shows an AFM topography image taken using a supersharp tip with nominal radius of curvature $\sim 1 \mathrm{~nm}$, of a typical region of the sample. The ferroelastic domains show up as a series of parallel lines oriented towards a common direction that in this case, varies from grain to grain, and have corresponding variations in height of approximately $0.3 \mathrm{~nm}$. For PFM imaging, Pt/Ir coated contact-mode cantilevers with nominal radius of curvature of the tip, $R=25 \mathrm{~nm}$, cantilever length, $L$, and stiffness, $k$, of $450 \mu \mathrm{m}$ and $0.08 \mathrm{Nm}^{-1}$, respectively, are used.

Figures 3 a,b \& c show the topography, magnitude and phase images of a PZT grain with (001) orientation, as can be seen by the fact that the polytwins are at either $45^{\circ}$ or $90^{\circ}$ to each other. From the magnitude and phase images we can see the parallel ferroelastic $a / a$ and $a / c$ domain stripes which are known as bundle domains. Some bundle domains are $c$-domain dominant, seen from the phase image as out-of-plane polarization (i.e. bright stripes) and some are $a$-domain dominant (i.e. dull stripes).

There are 4 types of ferroelastic domain wall available in PZT (001) as shown in Table 1 and Fig. 4. Using this information, we can then say that some of the polytwins are of the type $a / a$, whereas others are $a / c$. To determine which is which, we can make use of the fact that the regions with brightest contrast in the PFM magnitude image will be $c$-oriented, whereas the darkest will be $a_{1}$-oriented and perpendicular to the long axis of the cantilever, as this arrangement will not lead to an oscillation of the cantilever along the axis being detected. The corresponding arrangement of polytwins is shown in Fig. 5. The polarization cannot be uniquely determined in this way as we are not simultaneously measuring the lateral PFM signal, so there are 3 possibilities, oriented at $\pm 90^{\circ}$ or $180^{\circ}$ to that which we have suggested. In other words, it is not possible to determine whether a domain is $a_{1}{ }^{+}$or $a_{1}{ }^{-}$or $a_{2}{ }^{+}$or $a_{2}{ }^{-}$. We can however, determine the relative orientation of domains. 
From the contrast in the image, it is clear that region III must be of type $a-c$, and regions I and II that are at $\pm 45^{\circ}$ to this but at $90^{\circ}$ to each other must be of type $a_{1}-a_{2}$. It may also be seen that the $c$-regions (the brightest) in the magnitude image correspond to the regions in Fig. 3 (a) where the topography is slightly raised by around $2 \mathrm{~nm}$ compared to the surrounding area, which is also indicative of a $c$-oriented region surrounded by $a$-oriented regions. The sharpness of the domain walls in region I is due to the fact that they are perpendicular to the film plane, whereas the diffuse nature of the domain walls in region III is due to their $45^{\circ}$ tilt to the film plane. In regions I and II, the difference in magnitude between each pair of polytwins is the same, but the phase difference is $150^{\circ}$ and $0^{\circ}$, respectively (it is not $180^{\circ}$ due to the crosstalk between the piezoresponse and the Coulombic signals, demonstrating that the Coulomb force is playing a measureable role). The decreased resolution and contrast but increased brightness in both magnitude and phase in region II is due to the fact that adjacent polytwins are oscillating in the same direction, whereas in region I they are oscillating in anti-phase, so the resolution and contrast appear higher. The electric field extends laterally beyond the immediate tip-sample contact area, with a full width at halfmaximum of the field, $R_{E}$ of the order $40 \mathrm{~nm}$, so at any one point, the observed tip motion is due to the motion of the surrounding area of radius $R_{E}$.

\section{Experimental. II - Manipulating domains}

By using the tip to locally apply an electric field above the coercive value, we can change and manipulate the distribution of ferroelectric and ferroelastic domains. For (001) dominant PZT, depending on the type ( $a$ or $c$ ) of the polarization vectors, the switching angle of domains is expected to be either $45^{\circ}$ or $90^{\circ}$ away from the existing orientation. We carried out a poling cycle by scanning the area shown in Fig. 3 with a dc voltage of $-10 \mathrm{~V}$ followed 
by $+10 \mathrm{~V}$ applied between the cantilever tip and the bottom electrode. In Fig. 6(a), the resultant domain configuration is shown. The initial ferroelastic domains have changed from being aligned towards multiple directions to grow perpendicular to the nearest grain boundary, and apart from the central region which is $a_{1}-a_{2}$, and which remains unaltered, the $a-a$ domains have all rotated by $45^{\circ}$, indicating a change from $a-a$ to $a-c$ which is expected as the applied field is along the $z$ - direction. The reason for the non-switching of the central area is evidence that the switched polytwins have nucleated from the grain boundaries, and spread inwards - when they reach the central area, the polytwins there can either rotate by $+45^{\circ}$ or $45^{\circ}$, and the symmetric shape of this particular grain makes both equally favourable. This is evidenced in Fig. 6(b) after a further poling cycle, where it can be seen that the unswitched region has shrunk further inwards. After another poling cycle, this region has disappeared, and the polytwins have favoured orientation along the short axis of the grain, thus minimising the length and therefore the area of domain wall needed and hence the domain wall energy. After 6 poling cycles, as shown in Fig. 6(c), the polarisation has continued to evolve, and finally, after 10 poling cycles, no further changes are observed and the polarization configuration remains as shown in Fig. 6(d). The fact that the central polytwin remains unchanged throughout this process indicates a high density of planar defects in that region of the grain. This location coincides with the domain boundary between the $a-c$ and $a-a$ regions, where the domain walls change from being perpendicular to the film surface ( $a-a$ walls) to $45^{\circ}$ to the film surface ( $a-c$ walls). There are other regions of the grain where there is a similar change in wall morphology but which can be switched out by successive poling. This particular defect-rich region is therefore acting as a pinning site and is determining the evolution of polarization distribution for the entire grain.

As the polytwins in the central area of the grain shrink, this may also be seen in the simultaneously acquired topography images of Fig. 7. The images show the topography 
initially (Fig. 7(a)), after 1 poling cycle (Fig. 7(b)) and after 10 poling cycles (Fig. 7(c)). Zoom-ins of the magnitude and topography at the $a-a / a-c$ boundary are shown in Figs. (d), (f) and (e), (g), respectively. This region is typically $1 \mathrm{~nm}$ lower than the surrounding area, indicating $a_{1} / a_{2}$ domains surrounded by $c$-oriented regions. As successive poling cycles are carried out, the proportion of the grain that is $c$-oriented increases, although, as mentioned above, there is a region of planar defects that is slightly recessed (the grain is depressed by around $1 \mathrm{~nm}$ along that line), and show almost no PFM response, consistent with $a$ orientation. It can be seen that the whole grain has become buckled during this poling process as the grain has shifted from a significant proportion of $a$-polarization to almost entirely $c$-polarization. Height cross-sections of the grain initially, after 1 cycle and then after 10 cycles are shown in Figure 7(h). The difference in height between the centre and edge of the grain after each cycle is shown in figure 7(i) where it appears that after seven further cycles, the topography does not vary much anymore. This is something that we have observed many times, and origin of this effect is that as the grain evolves from $a$ to $c$ orientation, there is a corresponding deformation of the unit cell and the entire grain shrinks in diameter (laterally) by the proportional change in unit cell length from $c$ to $a$, which, for PZT is $2.6 \%$. We would therefore expect the film to increase in thickness by around $3.4 \mathrm{~nm}$ uniformly across the grain, while decreasing in lateral size by around $20 \mathrm{~nm}$. As the film is firmly attached to the substrate and therefore cannot shrink laterally at the base, a strain gradient throughout the film arises, and the top surface shrinks laterally while growing upwards, most freely at the edges. The net result of this is that the top surface becomes concave with a significantly larger change in topography of up to nearly $40 \mathrm{~nm}$. This is illustrated in Fig. 8(a) where we show the expected distribution of strain in the grain before and after the poling process. We start with the idealised assumption that the grain is strainfree and entirely $a$-oriented initially, and then upon poling, due to substrate clamping, some 
areas will remain $a$-oriented. The complex strain gradients associated with these isolated $a$ regions surrounded by $c$ regions will result in local deviations in the polarisation away from the expected directions via the flexoelectric effect. The combination of $a$ - and $c$-orientations result in bending of the surface, with a deformation as illustrated. It is also expected that the predominant polarisation of the surface is oriented along the surface normal with a number of isolated areas tilted away from the normal. These will appear either as small spots or extended lines of reduced PFM magnitude signal. This can explain the presence of the dark spots apparent in many of the PFM images presented here. An example is shown in Fig. 8 (b). These spots are typical of many of our experiments and are generally no smaller than around $20 \mathrm{~nm}$. These spots are often associated with a small variation in topography - typically a dip of around $0.2 \mathrm{~nm}$. In practice, due to the deposition process, the grains are pre-stressed, as evidenced by the multitude of ferroelastic domains that are seen. Therefore, there will be planes of defects (domain walls) starting at the substrate and extending through the entire film. In some cases, these defects will be so firmly anchored at the susbtrate/PZT interface that no amount of poling will remove them and relax out the stress. In these cases, these planar defects act as pinning sites and together with the local microstructure and grain geometry, determine the overall polarisation distribution and switching routes. This is a strong candidate for understanding what is happening in our experiments here. A direct consequence of this will of course be that should a device be fabricated at this lengthscale (i.e. on the order 1 micron or less), significant variations in polarisation distribution will occur initially, which will even out after a number of poling/switching cycles. However, there are cases when a spread in characteristics is seen even after many cycles, whose origin may well be down to the pinning observed here. Therein lies the double-edged sword of cheap and simple fabrication of sol-gel films over large areas, which is compounded by the inherent high density of defects. 


\section{Discussion}

In order to unravel the PFM data and to be able to determine the actual polarisation distributions, we need to understand what exactly we are measuring, which is the cantilever oscillation at the frequency $\omega$, due to the applied ac voltage. Here, we briefly consider the 4 mechanisms leading to this oscillation, look at their relative importance, and extract quantitative information regarding the sample under study.

1. Electrostriction. The sample deforms due to electrostriction - this is true of all dielectrics, is quadratic with applied field, and is described by

$$
z_{\text {electrostriction }}=Q t \varepsilon_{0}^{2}\left(\varepsilon_{r}-1\right)^{2}\left(E_{d c}+E_{a c} \sin \omega t\right)^{2}
$$

where $t$ is the film thickness, $Q$ is the electrostriction tensor and $\varepsilon_{r}$ is the relative permittivity of the sample. As PFM is concerned with measuring the component of cantilever deflection at the frequency, $\omega$, the relevant term is

$$
z_{\text {electrostriction }}{ }^{\omega}=2 t Q \varepsilon_{0}{ }^{2}\left(\varepsilon_{r}-1\right)^{2} E_{a c} E_{d c}
$$

In the absence of a dc bias voltage or contact potential difference between tip and sample, this term reduces to zero. However, when imaging ferroelectric samples using conducting AFM tips, there is always a small contact potential difference between the tip and the sample, on the order of 1$10 \mathrm{mV}$, which varies spatially across the sample. For typical experimental conditions, the resulting motion of the sample may be up to a few $10 \mathrm{~s}$ of $\mathrm{pm}$. 
This term depends directly on the dc bias applied between the tip and sample, so will contribute to any hysteresis measurements where the dc voltage is being swept.

\section{Local Coulomb interactions between the tip apex and the underlying} sample. The sample possesses a spontaneous polarization, $P_{S}$, which results in a surface (bound) charge density of magnitude $P_{S}$, and which produces an external electric field $E=P_{S} / 2 \varepsilon_{0}$. The voltage on the tip, $V_{t i p}$, causes the tip to become charged by an amount $Q_{t i p}=V_{t i p} C_{t i p}$, where $C_{t i p}$ is the tip-sample capacitance. There is a Coulombic interaction between this tip charge and the aforementioned Electric field from the sample, of magnitude

$$
F_{\text {Coul }}=Q_{\text {tip }} E=V_{\text {tip }} C_{\text {tip }} P_{S} / 2 \varepsilon_{0}
$$

Although the tip voltage is alternating in polarity, the sample polarization is not - it just oscillates about a mean value (assuming we are in the regime where the applied electric field is low enough or the frequency is high enough that the sample's polarization is not being switched), resulting in a force alternating between attractive and repulsive, i.e. at the frequency $\omega$. If the sample is polarized upwards, the surface will have a net positive charge, and when the tip is positively charged (i.e. is in the positive cycle of applied ac voltage), the electrostatic force will repel the tip away from the surface, so the tip will try to move upwards against the tip-surface adhesion force. If this Coulomb force is larger than the adhesion force, then the tip will lose contact 
with the surface for part of the oscillation cycle, and will be oscillating in an intermittent contact regime. The application of a voltage, $V$, which creates an electric field, $E$, between the tip and the surface causes the polarisation to be modified from $P_{S}$ by an amount $\Delta P=\varepsilon_{o}\left(\varepsilon_{r}-1\right) E$. The polarisation then becomes

$$
P=P_{S}+\varepsilon_{o}\left(\varepsilon_{r}-1\right) E
$$

As before, the total voltage between tip and sample is $V_{t i p}=V_{d c}+V_{a c} \sin \omega t$, and the applied electric field to first order is $E=V_{\text {tip }} / t$. Substituting in (4) above, we find the total Coulomb force between the tip and sample, at the frequency $\omega$ is given by:

$$
F_{\text {Coul }}^{\omega}=V_{a c} \frac{C_{t i p}}{2 \varepsilon_{o}} P_{S}+\frac{V_{d c} V_{a c} C_{t i p}\left(\varepsilon_{r}-1\right)}{t}
$$

The second term will show up as a constant offset to the measured force, except for the case of a hysteresis measurement where it will appear as a term linear in applied voltage (up to saturation). Both terms are similar in magnitude, and depend on the effective susceptibility and the relative magnitudes of $V_{d c}$ and $V_{a c}$.

The capacitance of an AFM tip above a dielectric surface has been addressed by several authors $[27,28]$ and is typically of the order 1-10 attoFarads. The resulting force can be of the order $10^{-7} \mathrm{~N}$, but due to screening under ambient conditions, it is reduced to $10^{-10}-10^{-9} \mathrm{~N}$. 
3. Piezoelectric deformation. An electric field $E$, applied to a piezoelectric material with polarization $P_{S}$ will cause deformation of the sample in directions and by amounts determined by the crystal properties and symmetry, information that is encapsulated in the piezoelectric tensor $d_{i j}$. For PZT, if $E$ is oriented parallel to $P_{S}$, the sample will expand. For example, to compare with the Coulomb force, if the sample is polarized upwards, then a positive voltage on the tip will lead to a downwards-oriented electric field, and therefore downwards motion of the tip (contraction of the sample), in the opposite direction to the Coulomb-induced force. For the case of (001) - oriented PZT, the applied electric field, $E_{\text {tot }}$, creates a piezoelectric deformation, $z_{\text {piezo }}=d_{33} E_{\text {tot }} t=d_{33} t\left(E_{c}+E \sin \omega t\right)$. Again, we are only interested in the term at the frequency $\omega$, so the relevant piezoelectric deformation of the sample is

$$
z_{\text {piezo }}{ }^{\omega}=\int_{z=0}^{t} d_{33} E(z) d z=d_{33} V
$$

To determine the magnitude of the electric field, to first order, it is approximately $V / t$ (as the integral of $V$ across the film must equal $E t$ ), so the deformation $z_{\text {piezo }}{ }^{\omega}=d_{33} V$. For PZT, $d_{33} \sim 50 \mathrm{pm} \mathrm{V}^{-1}$, so for an applied voltage of $6 \mathrm{~V}$, we would expect a deformation of the order $300 \mathrm{pm}$. In reality, the field is non-uniform and decays rather rapidly with distance, so the effective area being sampled is typically comparable to or less than the tip radius. The electric field drops to $5 \%$ of the maximum value around halfway through the film. It must also be noted that the actual potential that the sample sees depends very sensitively on the nature of the tip-sample contact, due to 
dead layers on the sample, contaminants on the surface, the wearing of the metal coating on the tip apex and the level of indentation of the surface, the latter of which depends on the imaging conditions and the tip-sample forces. It is therefore challenging to know the exact field that the sample experiences, so there will be cases in which truly quantitative PFM imaging is impossible. Nonetheless, PFM has its place in the study of piezoelectric materials due to the information we can obtain about the distribution of domains, the effect of topography, and the ability to manipulate domains using the tip field.

4. Cantilever buckling. There is a non-local electrostatic force acting over the body of the entire cantilever, which, neglecting any relative tilt between the cantilever and the underlying surface is of magnitude

$$
F_{\text {non-local }}=\varepsilon_{o} \frac{S\left(V_{d c}+V_{a c} \sin \omega t\right)^{2}}{2 T}
$$

where $S$ is the cantilever area (Length $\times$ width) and $T$ is the tip length. In the absence of any dc potential difference between the tip and the sample, this force is zero at $\omega$, but if the tip-sample voltage has a dc component, then the resulting force at $\omega$ is

$$
F_{\text {non-local }}^{\omega}=\varepsilon_{o} \frac{S V_{d c} V_{a c}}{T}
$$

which is typically orders of magnitude weaker than the local Coulomb term. The associated measured angular deflection of the cantilever is a complex 
function of position, and is zero at the centre and both ends, so the relative importance of this term will depend on the exact position of the laser spot.

There is however another, much more significant source of cantilever buckling, which is due to the in-plane components of sample polarization along the cantilever axis leading to shearing of the top surface by an amount $\delta y$, that is proportional to $d_{31} E$. As with the other contributions above, this has been dealt with in detail elsewhere $[29,30]$, but we will summarize the main points here, as relevant to our particular experimental setup. Consider the top view of a cantilever, as shown in Fig. 9. We have also shown some (001) polytwins at an angle $\varphi$, to the cantilever axis. Using the coordinate system shown here, on an $a^{+}{ }_{1}$-domain, the projection of polarization, $P_{S}$, along the cantilever axis is $-P_{S} \cos \left(\frac{\pi}{4}+\varphi\right)$. For an ${a_{1}^{-}}_{1}$ domain (which is in the opposite sense to the $a^{+}{ }_{1}$-domain), the component is $P_{S} \cos \left(\frac{\pi}{4}+\varphi\right)$. Likewise, on an $a^{+}{ }_{2}$-domain, the projection of polarization along the cantilever axis is $P_{S} \sin \left(\frac{3 \pi}{4}+\varphi\right)=P_{S} \cos \left(\frac{\pi}{4}+\varphi\right)$. For an $a_{2}^{-}$domain (which is in the opposite sense to the $a_{2}^{+}$-domain), the component is $-P_{S} \cos \left(\frac{\pi}{4}+\varphi\right)$. Therefore, for the case where the cantilever is oriented at $\pi / 4$ to the polytwins, if it is on an $a_{1}$ domain, then there will be no piezoresponse. If it is on an $a_{2}$ domain, then as the top surface shears, as long as the amplitude or frequency are not so large that the tip slides, then the tip will move along with the surface and the cantilever will buckle. There is no change in tip height associated with this, but there is a change in the cantilever angle, which is indistinguishable from a vertical deflection. 
We can now combine the above factors to estimate the tip motion for the different scenarios encountered during a typical PFM experiment, bearing in mind that the optical beam-deflection system used in the vast majority of AFMs measures the cantilever angular deflection rather than vertical displacement. The models considered above are all assuming that there is negligible indentation of the sample by the tip, so are particularly appropriate for the case where the cantilever stiffness is small and the tip-sample forces are low. This is the reason for our choice of soft cantilevers (i.e. ones with stiffness $<1 \mathrm{~N} / \mathrm{m}$ ).

On a $c$-domain, the expected deflection will be the difference between the piezoelectric deformation of the sample and that due to the Coulomb force:

$$
z_{c}=d_{33} V-F_{\text {Coul }} / k
$$

Solving the beam equation $[31,32]$ for a cantilever of length $L$, Young's modulus $Y$ and moment of inertia $I$ subjected to a normal force $F$ at its end gives the deflection at the tip end as:

$$
Z_{c}=\frac{F}{Y I} \times\left(\frac{L^{3}}{3}\right)
$$

and an angular deflection of

$$
\theta=\frac{F}{E I} \times\left(\frac{L^{2}}{2}\right)
$$


As the tip is in contact with the sample, when the surface shears laterally, this leads to buckling of the cantilever. The lateral deflection of the tip by an amount $\delta y$ causes a change in the cantilever angle $\delta \theta=\tan ^{-1}\left(\frac{\delta y}{T}\right)$. This is the same angle that is produced by a vertical deflection $z^{\prime}{ }_{c}$ where $z^{\prime}{ }_{c}=\frac{2 L \delta \theta}{3}=\frac{2 L}{3} \tan ^{-1}\left(\frac{\delta y}{T}\right)$. Given that $\delta y<<T$, we can make the approximation $\tan ^{-1}\left(\frac{\delta y}{T}\right) \sim\left(\frac{\delta y}{T}\right)$, so although the deflection angle of the cantilever end is $\frac{\delta y}{T}$ this is the same angle that is caused by a vertical deflection of $\frac{2 L}{3} \frac{\delta y}{T}$, and the two are indistinguishable.

We can therefore describe the measured signal in the AFM as being the vertical deflection times $3 / 2 L$, scaled by the characteristics of the photodiode, laser, lock-in amplifier and associated electronics. We will call the scaling factor $A$. For our microscope and the cantilevers used in these experiments, $A=10^{-6}$ Amps per degree of angular deflection. As the cantilevers are driven near resonance, the angular deflection is increased by the effective $Q$ factor, $Q^{\prime}$, which, in our experiments is typically of order 10 .

Combining the above information, the measured signal in vertical PFM on a $c$ domain for the case of an ac voltage, $V_{a c}$ applied between tip and sample is

$$
P R_{v}=\frac{3 A Q^{\prime}}{2 L} V_{a c}\left(d_{33}-\frac{C_{t i p}}{k}\left[\frac{P_{S}}{2 \varepsilon_{o}}+\frac{V_{d c}\left(\varepsilon_{r}-1\right)}{t}\right]\right)
$$

In order for this approach to be quantitative, the cantilever stiffness, $k$, must be measured using any of the techniques that are in common use [33].

The last term can be eliminated if the contact potential difference is monitored and maintained at $0 \mathrm{~V}$. It should also be noted that the relative magnitudes of the different terms 
also depend on the position of the laser spot on the cantilever. Additionally, as long as the Coulomb force is lower than the adhesion force of the cantilever, it will not contribute to the measured deflection. As either the dc or ac voltage, or both, are increased, we will enter a regime where the Coulomb force is larger than the adhesion force. At this point, the upper limit on the cantilever deflection will be given by equation (12). For this reason, it is not possible to obtain fully quantitative information using PFM when Coulomb forces are significant. Nonetheless, it is borne out by experiment that PFM images with better contrast can be obtained when operating in the regime where Coulomb forces are of the same order of magnitude as the adhesion force.

On an $a^{+}{ }_{1}$ domain, the measured signal will be:

$$
P R_{a 1}=\frac{d_{31} V A}{T} \cos \left(\frac{\pi}{4}+\varphi\right)
$$

Whereas on an $a^{+}{ }_{2}$ domain, the measured signal will be:

$$
P R_{a 2}=\frac{d_{31} V A}{T} \sin \left(\frac{\pi}{4}+\varphi\right)
$$

It should be noted that we anticipate there will be no Coulomb force when imaging in-plane (a) polarized regions, apart from at grain boundaries, which may be charged.

Overall, having considered these contributions to the piezoresponse signal, the mimimum expected deflection of the cantilever will occur when the cantilever is at $45^{\circ}$ to the polytwins, with the tip on an $a_{1}$ domain where there is no component of polarization along the cantilever axis, and no Coulomb force. When the tip is on $c$ regions, the local Coulombic interaction will play a role, and will vary depending on the position on the surface. As the voltage is increased, this interaction increases, and when it is large enough, the force becomes sufficiently larger than the adhesion force and the tip may start to leave the surface. This 
intermittent-contact regime is very sensitive to local variations in the polarization and contact stiffness and cannot therefore be used for quantitative measurements of polarization or phase except in specific circumstances such as those presented in this paper, as the capacitive signal dominates and obscures the true piezoresponse. To clarify, in this regime where the Coulomb force produces a significant angular deflection of the cantilever, it is variations in the distribution of surface charge that are being imaged rather than the bulk polarization, but as these are intimately related, we can qualitatively consider them to be the same. A detailed knowledge of the factors leading to motion of the cantilever can be used to predict the expected signal levels in a PFM experiment. We will apply the above principles to the results presented earlier to facilitate image interpretation and to better enable the polarization to be inferred.

In region I of Fig. 5, the angle between the polytwins and the cantilever axis, $\varphi=16^{\circ}$. Given the geometry of the cantilever and the magnitude of ac voltage (6V) applied to the tip, we expect the piezoresponse signal on an $a_{1}$ domain to be approximately $1.6 \mathrm{pA}$, and on an $a_{2}$ domain to be $3.3 \mathrm{pA}$. However, we find that the piezoresponse varies between around $1.5 \mathrm{pA}$ and $6 \mathrm{pA}$. By contrast, on a $c$ region (region III), if the Coulomb force is negligible, we expect the signal to be of the order $8 \mathrm{pA}$, which is less than half the measured value of $18 \mathrm{pA}$. Therefore, we can conclude that a significant proportion of the measured piezoresponse whilst imaging $c$-domains is due to the local Coulomb force on the tip. Assuming that the dc contact potential difference is negligible, the final term in the vertical piezoresponse (equation (12)) is much smaller than the middle term, which predicts a PFM signal of the order $100 \mathrm{nA}$, which is over 4000 times larger than what is actually observed. This discrepancy is due to screening of the surface bound charge by surface species, including hydrocarbons and water, primarily via hydrolysis [34-36]. The tip-surface Coulomb force, from equation (5) is of the order $10^{-11} \mathrm{~N}$, considerably less than the tip-sample adhesion force, 
indicating that the tip remains in contact with the surface even under resonant oscillation. It must be pointed out that quantitative measurements are of course difficult to perform as the tip-sample contact stiffness varies during scanning, with the consequence that the resonance frequency is not constant, and therefore the effective Q-factor is not constant. The calculations here are merely intended to serve as a guide. In order to be able to obtain quantitative information, (i) the cantilever should be oscillated far from any in-contact resonances and (ii) the cantilever deflection should be simultaneously measured at $\omega$ and $2 \omega$. If pure piezoresponse data is required, then the Coulombic terms should be at least 100 times smaller than for our experiments, necessitating the use of cantilevers with normal spring constants of the order $7 \mathrm{Nm}^{-1}$ or above. However, this then leads to a regime where the tip will be indenting the sample, complicating image interpretation and reducing the resolution. A point to note is that despite the expected variations in effective Q-factor mentioned above, it is possible to obtain images where the contrast between domains is stable and remains constant over the entire image, suggesting that in many cases, on similarly-oriented domains, the cantilever oscillation is stable. This stability is what leads us to suggest that it is possible to obtain at least semi-quantitative information in this mode of operation.

Now we should address the discrepancy between the expected and measured cantilever deflections for in-plane piezoresponse. As stated earlier, we would expect to see no Coulombic deflection of the cantilever when on an in-plane polarized region, as in this case, there is no surface charge, apart from possibly at domain walls. The difference we find between model and experiment is not a simple ratio, indicating that even if our value of $d_{31}$ is inaccurate, we cannot account for the discrepancy, as the ratio of piezoresponse signals on an $a_{2}$ to an $a_{1}$ region is independent of $d_{31}$, and we find it to be 1.8 instead of the expected 4.6. There are three further factors we need to consider: (i) the lateral force associated with the tip-surface contact, (ii) the possibility that the distribution of polarization within $a_{1}$ and $a_{2}$ 
regions deviates from expectations and (iii) the interaction volume experiencing a significant electric field is large enough to include more than one stripe, so we are in essence sampling an average across multiple stripes, which would account for the fact that the ratio of signals is lower than expected. In region I, the width of the polytwins is around $15-20 \mathrm{~nm}$, which is smaller than the extent of the electric field, so several polytwins will be contributing to the overall measured piezoresponse. If the orientation relative to the coordinate system we have shown is such that the polytwins in region I are $a_{1} /-a_{2}$ or $-a_{1} / a_{2}$, then we would expect the piezoresponse signal to be greater, and up to around $5 \mathrm{pA}$ on the $a_{2}$ regions, and larger than $1.6 \mathrm{pA}$ on the $a_{1}$ regions, increasing the ratio of measured signals towards the expected value, but never reaching it. This simply highlights the fact that we cannot perform quantitative measurements on polytwins that are smaller than a critical size, determined by the extent of the applied electric field.

Given the lateral displacement of the surface of around $60 \mathrm{pm}$, we expect the lateral force on the tip to be of the order $90 \mathrm{pN}$. This force is small enough that it is reasonable to expect the tip to remain in contact with the same point on the surface and there is little or no sliding associated with the piezoelectric-induced motion of the tip on the sample surface, so this is expected to have no effect on the measured signal, and therefore is not a reason for the departure from the expected behaviour.

The other issue we have raised is the possibility that we are not imaging the in-plane domains in the way we expect. Two possibilities are (i) due to the flexoelectric effect, the inplane polarization has been distorted and has a small component out of plane and (ii), at the $a_{1}-a_{2}$ domain walls, there is some charge accumulation, which may even be due to some head-to head polarized regions. In any case, this would lead to a Coulomb force that would account for the increased signal amplitudes. Earlier measurements on vertical PFM with stiffer cantilevers showed very good agreement with expectations, indicating that the 
differences we are observing are due to the shift towards more dominant Coulomb forces, but only further experimentation will help to untangle this issue. As regards the flexoelectric effect, the electric field that points out of plane due to a vertical strain gradient $u$ ' is

$$
E_{\text {flexo }}=\frac{e}{4 \pi \varepsilon_{o} a} u^{\prime}
$$

where $e$ is the electron charge and $a$ is the in-plane lattice constant. Given the numerical value of the prefactors, in order for $E_{f l e x o}$ to be comparable to the applied field, i.e. $10^{7} \mathrm{Vm}^{-1}$, then the strain gradient would need to be of the order $3 \times 10^{6} \mathrm{~m}^{-1}$. Such strain gradients have been reported [37] in similar systems, so this is a possibility that should be explored in future experiments.

For comparison and as a validation step, another region of the sample is shown in Fig. 10, where the polytwins are now at $45^{\circ}$ to the cantilever axis. These polytwins are wider than in the previous case, and are in fact mostly wider than the effective extent of the electric field from the tip, so when on an $a_{1}$ domain, the PFM signal does now go to zero, indicating that (i) the deformation due to electrostriction is indeed negligible so for most cases can be ignored and (ii) there are no other factors leading to displacement of the cantilever. In regions where the $a_{1}$ domain is narrower, the field extends into the neighbouring $a_{2}$ (or $c$ ) region, and there is a small PFM signal. When the $a_{1}$ region has a half-width larger than around $25 \mathrm{~nm}$, the PFM signal drops to zero, indicating that the effective area being probed by the tip when away from any domain walls is of the order $20-25 \mathrm{~nm}$, comparable to the tip radius. When on the neighbouring regions, the PFM signal rises to a maximum of just over $3.7 \mathrm{pA}$, which is too small to be a $c$-oriented region. On the basis of equation (14), if this is an $a_{2}$-region, we would expect a PFM signal of approximately $3.7 \mathrm{pA}$, in excellent agreement with our findings. It would appear therefore that there are cases in which quantitative PFM is indeed 
possible, especially when imaging in-plane components of polarization which produce buckling of the cantilever along it's long axis. It is out-of-plane PFM with more complex factors determining the cantilever motion that is more challenging to quantify.

\section{Summary}

We investigated image formation and contrast in piezoresponse force microscopy of thin-film lead zirconate titanate, with the cantilever operating in resonant mode. We have shown that even in this mode, and when using a "soft" cantilever, although the local Coulomb force on the tip may be significant, it is still possible to obtain quantitative estimates of the surface polarization, the degree of surface screening, and relative orientation of domains using a simple analytical model.. The response of a region of the film to an applied electric field large enough to induce switching was then analysed, and the role of defects was explored. We found that the top surface of grains initially containing a large proportion of in-plane polarised domains deformed into a concave shape after poling modified the polarisation to be mostly out-of-plane. This buckling deformation is significant, and results from substrate clamping. 


\section{REFERENCES}

1. R. Ramesh \& D. G. Schlom, Science 296 (5575), 1975 (2002)

2. S. Ducharme \& A. Gruverman, Nat. Mat. 8 (1), 9 (2009)

3. N. Setter, D. Damjanovic, L. Eng, G. Fox, S. Gevorgian, S. Hong, A. Kingon, H. Kohlstedt, N. Y. Park, G. B. Stephenson, I. Stolitchnov, A. K. Tagantsev, D. V. Taylor, T. Yamada \& S. Streiffer, J. Appl. Phys. 100 (5) (2006)

4. C. L. Jia, K. W. Urban, M. Alexe et al, Science 331 (6023), 1420 (2011)

5. C. L. Jia, S. B. Mi, K. Urban et al, Nat. Mat. 7, 57 (2008)

6. P. D. Nellist, M. F. Chisholm, N. Dellby et al, Science 305 (2004)

7. J. Seidel, L. W. Martin, Q. He et al, Nat. Mat. 8 (3), 229 (2009)

8. J. Wang, J. B. Neaton, H. Zheng et al, Science 299 (5613), 1719 (2003)

9. R. K. Vasudevan, W. Wu, J. R. Guest, A. P. Baddorf, A. N. Morozovska, E. A. Eliseev, N. Balke, V. Nagarajan, P. Maksymovych \& S. V. Kalinin, Adv. Funct. Mater. 23 (20), 2592 (2013)

10. S. V. Kalinin, A. Rar \& S. Jesse, IEEE Tuff, (2013)

11. Y. Ivry, D. P. Chu \& C. Durkan, Appl. Phys. Lett. 94, 162903 (2009)

12. P. Güthner \& K. Dransfeld, Appl. Phys. Lett. 61, 9 (1992)

13. K. Franke, J. Besold, W. Haessler \& C. Seegebarth, Surf. Sci., 302 L283 (1994)

14. T. Hidaka, T. Maruyama, M. Saitoh, N. Mikoshiba, T. Shiosaki, L. A. Wills, R. Hiskes, S. A. Dicarolis \& Jun Amano, Appl. Phys. Lett. 68, 2358 (1996)

15. A. Gruverman, H. Tokumoto, A. S. Prakash, S. Aggarwal, B. Yang, M. Wuttig, R. Ramesh, O. Auciello \& T. Venkatesan, Appl. Phys. Lett. 71, 3492 (1997)

16. G. Zavala, J. H. Fendler \& S. Trollier-McKinstry, J. Appl. Phys. 81, 7480 (1997)

17. C. Harnagea, A. Pignolet, M. Alexe, D. Hesse \& U. Gösele Appl. Phys. A 70, 261 (2000) 
18. A. Garcia-Melendrez \& C. Durkan, Appl. Phys. Lett. 103, 092904 (2014)

19. H. Shin, J. K. Shin, S. Hong, J. U. Jeon, H. W. Song, J. I. Hong \& K. No, Integ. Ferroelectr. 38, 31 (2001)

20. A. Gruverman \& S. V. Kalinin, J. Mater. Sci. 41, 107 (2006)

21. E. Soergel, J. Phys. D: Appl. Phys. 44, 464003 (2011)

22. S. V. Kalinin, B. J. Rodrigues, S. Jesse, J. Shin, A. P. Baddorf, P. Gupta, H. Jain, D. B. Williams \& A. Gruverman, Microsc. \& Microan. 12, 206 (2006)

23. F. Peter, A. Rüdiger, R. Waser, K. Szot \& B. Reichenberg, Rev. Sci. Inst. 76, 046101 (2005)

24. S. Jesse, A. P. Baddorf \& S. V. Kalinin, Nanotechnology 17, 1615 (2006)

25. Y. Ivry, N. Wang, D. P. Chu \& C. Durkan, Phys. Rev. B 81, 174118 (2010)

26. G. Catalan, L. J. Sinnamon \& J. M. Gregg, J. Phys.-Condens. Mat. 16, 2253 (2004)

27. G. Gramse, I. Casuso, J. Toset, L. Fumagalli \& G. Gomila, Nanotechnology 20, $395702(2009)$

28. C. Durkan, M. E. Welland, D. P. Chu \& P. Migliorato, Phys. Rev. B. 60, 16198 (1999)

29. C. Harnagea, A. Pignolet, M. Alexe \& H. Hesse, Integr. Ferroelectr. 38, 667 (2001)

30. E. Bonnacurso, F. Schönfeld \& H-J. Butt, Phys. Rev. B 74, 085413 (2006)

31. T. L. Horng, J. Solid. Mech. \& Mat. Eng. 1, 1063 (2007) doi: 10.1299/jmmp.1.1063

32. G. Y. Chen, R. J. Warmack, T. Thundat \& D. P. Allison, Rev. Sci. Instrum. 65, 2532 (1994)

33. C. Rawlings \& C. Durkan, Nanotechnology 23, 485708 (2012)

34. J. E. Sader, J. W. M. Chon \& P. Mulvaney, Rev. Sci. Instrum. 70, 3967 (1999)

35. S. M. Cook, T. E. Schaffer, K. M. Chynoweth, M. Wigton, R. W. Simmonds \& K. M. Lang, Nanotechnology 17, 2135 (2006) 
36. P. Zubko, G. Catalan, A. Buckley, P. R. L. Welche \& J. F. Scott, Phys. Rev. Lett. 99, 167601 (2007) 


\section{FIGURE CAPTIONS}

Fig. 1. Schematic showing the experimental set-up for PFM. An AC (with the option of adding a DC) voltage source at frequency $\omega$ is applied to the tip with the sample grounded, and the corresponding motion of the cantilever at $\omega$ is measured.

Fig. 2. High-resolution AFM image $(2.5 \mu \mathrm{m} \times 2.5 \mu \mathrm{m})$ taken using a tip with nominal radius of curvature $1 \mathrm{~nm}$, where polytwins are evident across multiple grains. The polytwins appear as a series of parallel lines in the topography with corresponding variations in height of approx. $0.3 \mathrm{~nm}$. The scale bar is $500 \mathrm{~nm}$.

Fig. 3. (a) topography, (b) magnitude and (c) phase images of an area of polycrystalline PZT $1.2 \mu \mathrm{m} \times 1.2 \mu \mathrm{m}$ across showing a complex arrangement of polytwins at $45^{\circ}$ and $90^{\circ}$ to each other, consistent with (001) orientation. Scale bar is $400 \mathrm{~nm}$.

Fig. 4. Top views of (a) Possible domain walls of type $c / a_{1}$, (b) possible domain walls of type $c / a_{2}$ and (c) side view of type $c / a$ walls. $a-a$ walls are vertical to the film surface as opposed to $c / a$ walls that are at $45^{\circ}$ to the surface.

Fig. 5. Zoom-in of magnitude image from Fig. 3(b), size $750 \mathrm{~nm} \times 530 \mathrm{~nm}$. The scale bar is $200 \mathrm{~nm}$. The polytwins are split into three distinct regions, each with a different orientation and contrast. The orientation of the in-plane polarization vectors, $\mathrm{a}_{1}$ and $\mathrm{a}_{2}$ are shown as white arrows, and the projection of the polarization vectors parallel to the long axis of the cantilever are shown as black arrows. Regions I and II contain $a$ - $a$ polytwins whereas region III contains $a-c$ polytwins.

Fig. 6. PFM magnitude images after (a) 1, (b) 2, (c) 6 and (d) 10 poling cycles of $-10 \mathrm{~V} \mathrm{dc}$ applied to the AFM tip during scanning followed by $+10 \mathrm{~V}$. The central $a_{1}$ - $a_{2}$ region shrinks 
after each poling cycle until eventually there are just a few polytwins remaining. The largest of these is at the location of the original domain boundary between a-a and a-c polarized regions. The scale bar in all images is $400 \mathrm{~nm}$.

Fig. 7. Topography images of (a) the original state, (b) after one poling cycle and (c) after 10 poling cycles of the grain. The central $a$ - $a$ regions appear as a dip in the topography, as does the line/plane of defects at the $a-a$ and $a-c$ boundary which remains throughout the poling and is seen as a pinning site. Zoom-in of the PFM magnitude (d), (f) and topography (e), (g) at the $a-a / a-c$ boundary before the cycles and after 10 cycles, respectively. The height scale in (e) is $1.5 \mathrm{~nm}$. Cross-sections in (h) show how the top surface of the grain rises and changes from being relatively flat to concave to compensate for the change in geometry associated with switching from mostly a-oriented to mostly c-oriented. In (i), we show the changes in height at the centre of the grain as the poling cycles progress. The scale bars are $400 \mathrm{~nm}$ in (a)-(c), $150 \mathrm{~nm}$ in (d)-(e) and $160 \mathrm{~nm}$ in (f)-(g).

Fig. 8. (a) Illustration of the side-view of the grain before (top) and after (bottom) poling by the AFM tip. We show the grain as being $a$-oriented before poling and mostly $c$-oriented after. Due to the substrate clamping, the entire grain cannot switch to $c$-orientation, and there will therefore be regions that remain $a$-oriented. The resulting strain gradient will impose a rotation away from both of these directions in a small number of unit cells. (b) PFM image of a $400 \mathrm{~nm} \times 200 \mathrm{~nm}$ area showing spots $\sim 20 \mathrm{~nm}$ across - regions that are not $c$-oriented. Scale bar $=50 \mathrm{~nm}$.

Fig. 9. The AFM cantilever is at an angle, $\varphi$ to the polytwins, whose polarization vectors $a_{1}$ and $a_{2}$ (which are at $45^{\circ}$ to the polytwins) can be decomposed into components along and perpendicular to the cantilever axis. 
Fig. 10. (a) PFM magnitude of a different grain on the same sample, but where the polytwins are oriented at $45^{\circ}$ to the cantilever axis. The lowest signal is $0 \mathrm{pA}$, corresponding to $a_{1}$ orientation, and the largest is $3.7 \mathrm{pA}$, corresponding to $a_{2}$ orientation. The PFM signal profile taken along the line indicated in (a) is shown in (b). Scale bar $=150 \mathrm{~nm}$. 


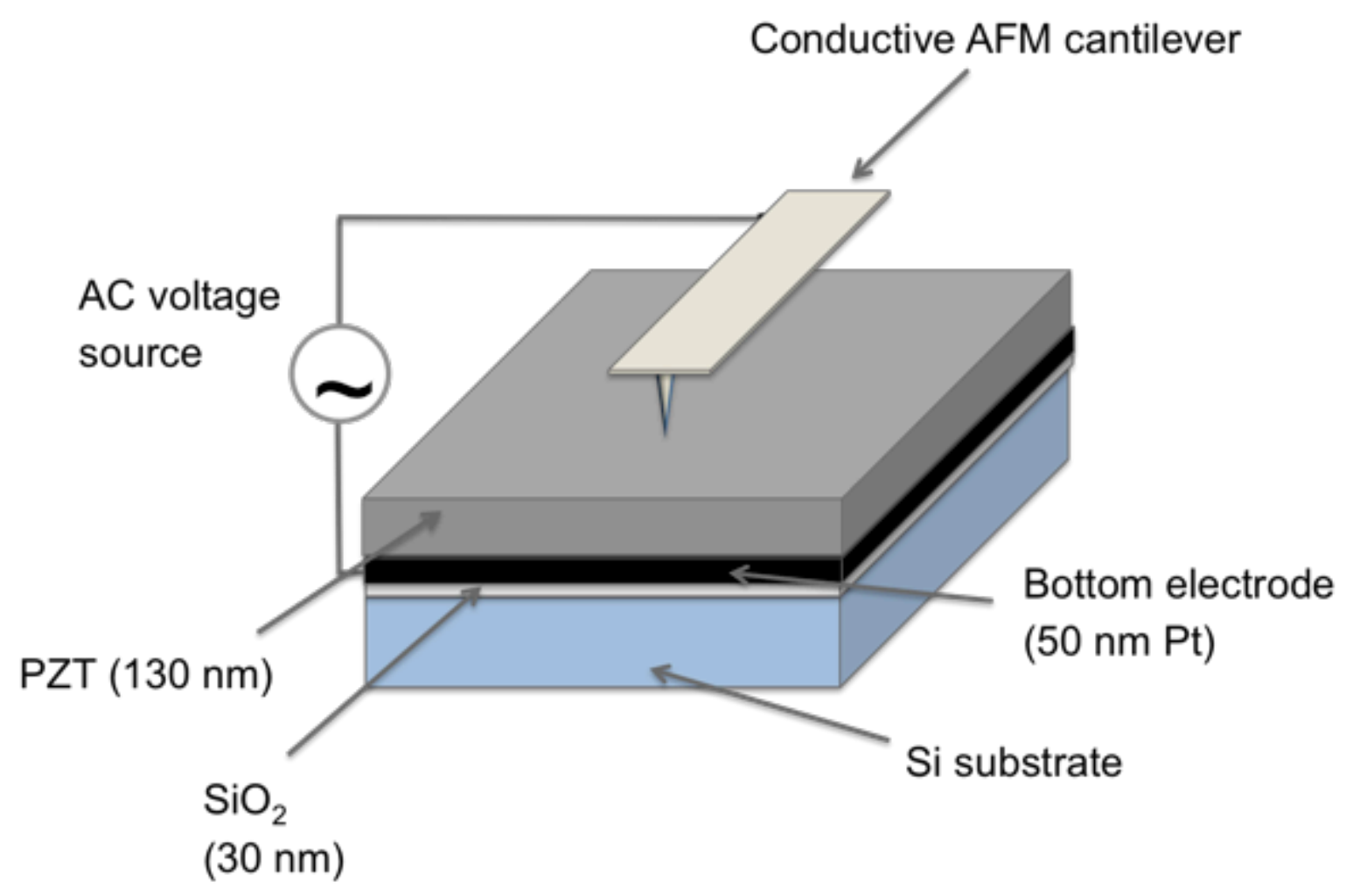

Fig. 1 


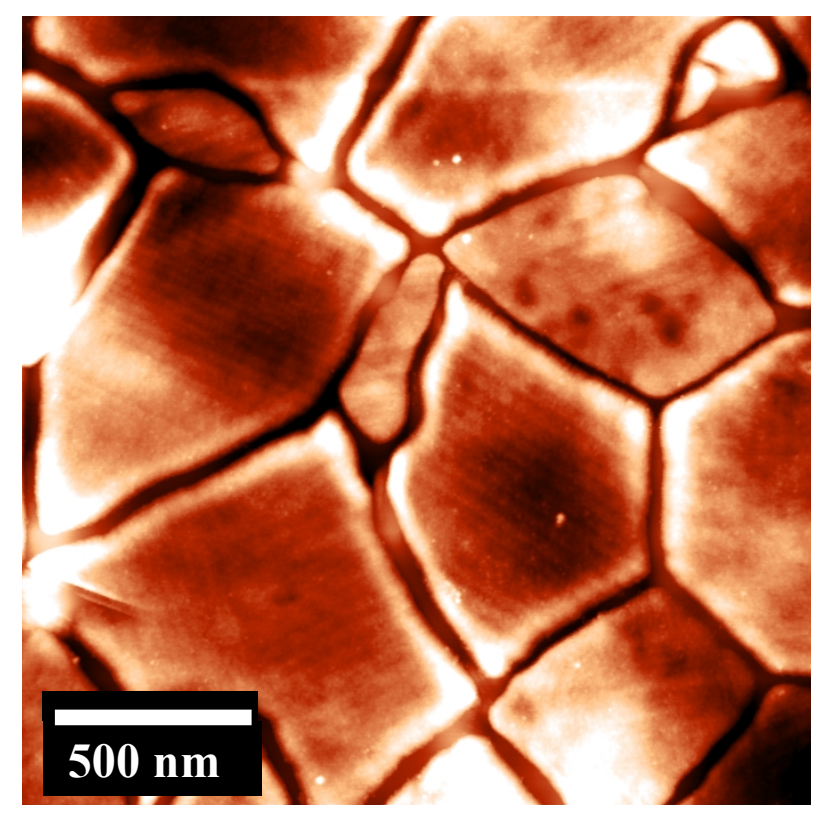

Fig. 2 

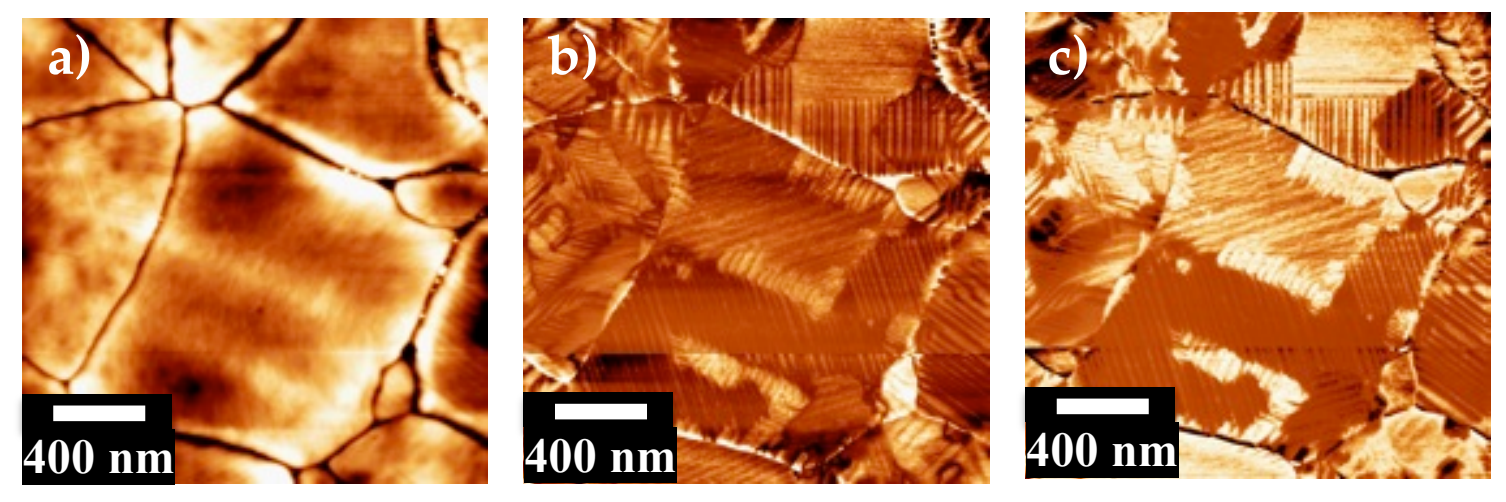

Fig. 3 
Table 1

\begin{tabular}{|l|l|l|l|l|}
\hline & \multicolumn{4}{|c|}{ Polarization vectors } \\
\hline Type I & $\vec{c}^{+} / \vec{a}_{1}^{+}$ & $\vec{c}^{+} / \vec{a}_{1}^{-}$ & $\vec{c}^{-} / \vec{a}_{1}^{+}$ & $\vec{c}^{-} / \vec{a}_{1}^{-}$ \\
\hline Type II & $\vec{c}^{+} / \vec{a}_{2}^{+}$ & $\vec{c}^{+} / \vec{a}_{2}^{-}$ & $\vec{c}^{-} / \vec{a}_{2}^{+}$ & $\vec{c}^{-} / \vec{a}_{2}^{-}$ \\
\hline $\begin{array}{l}\text { Type IIIa } \\
\text { Type IIIb }\end{array}$ & $\vec{a}_{1}^{+} / \vec{a}_{2}^{+}$ & $\vec{a}_{1}^{-} / \vec{a}_{2}^{-}$ & & \\
\hline \hline
\end{tabular}

Relative angle

Type I $\angle$ Type II $=90^{\circ}$

Type I $\angle$ Type IIIa $=45^{\circ}$

Type I $\angle$ Type IIIb $=-45^{\circ}$

Type II $\angle$ Type IIIa $=-45^{\circ}$

Type II $\angle$ Type IIIb $=45^{\circ}$

Table 1. The different types of domain walls in PZT (001), and the relative angles between them. 
a)

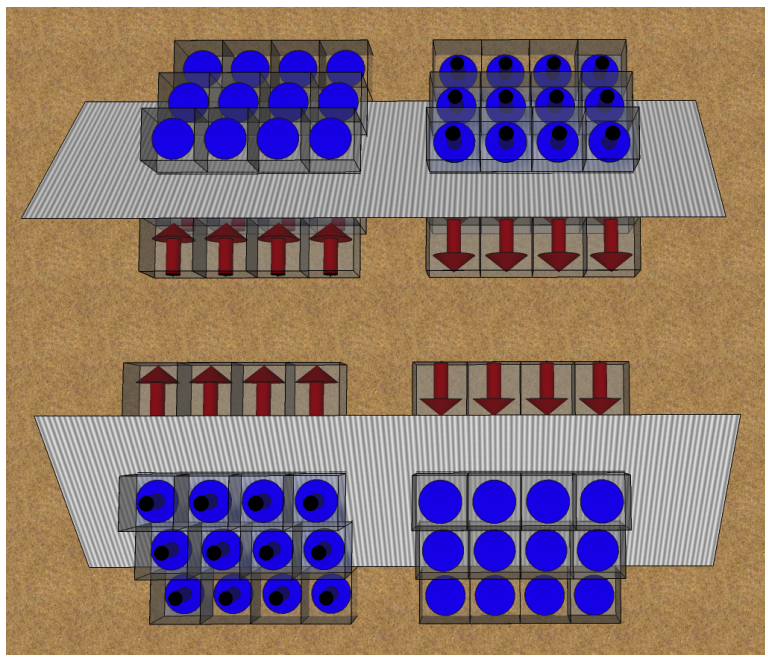

b)

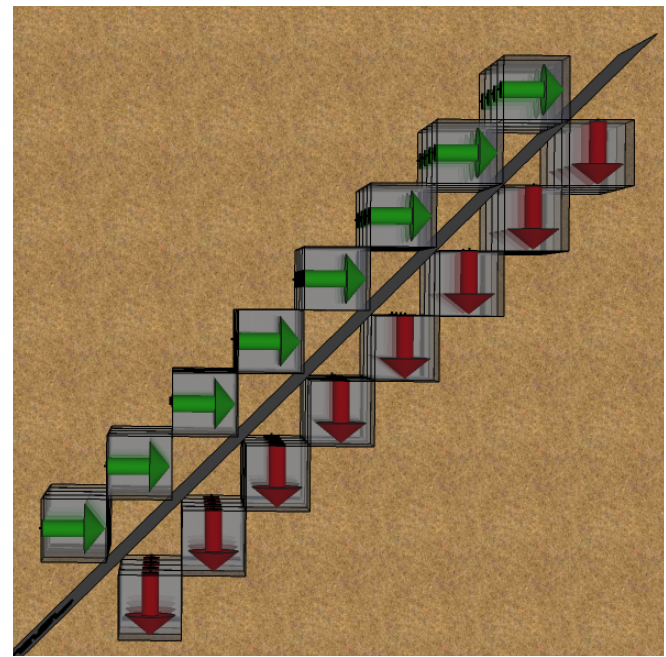

c)

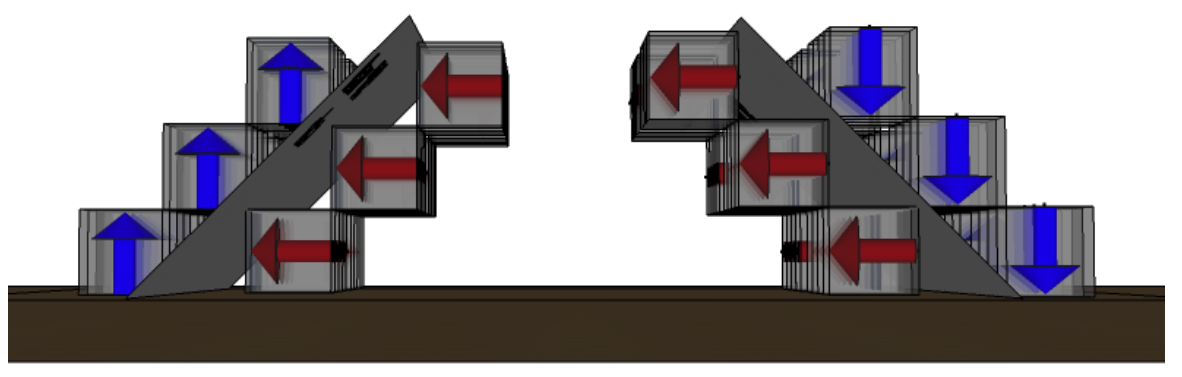

Fig. 4 

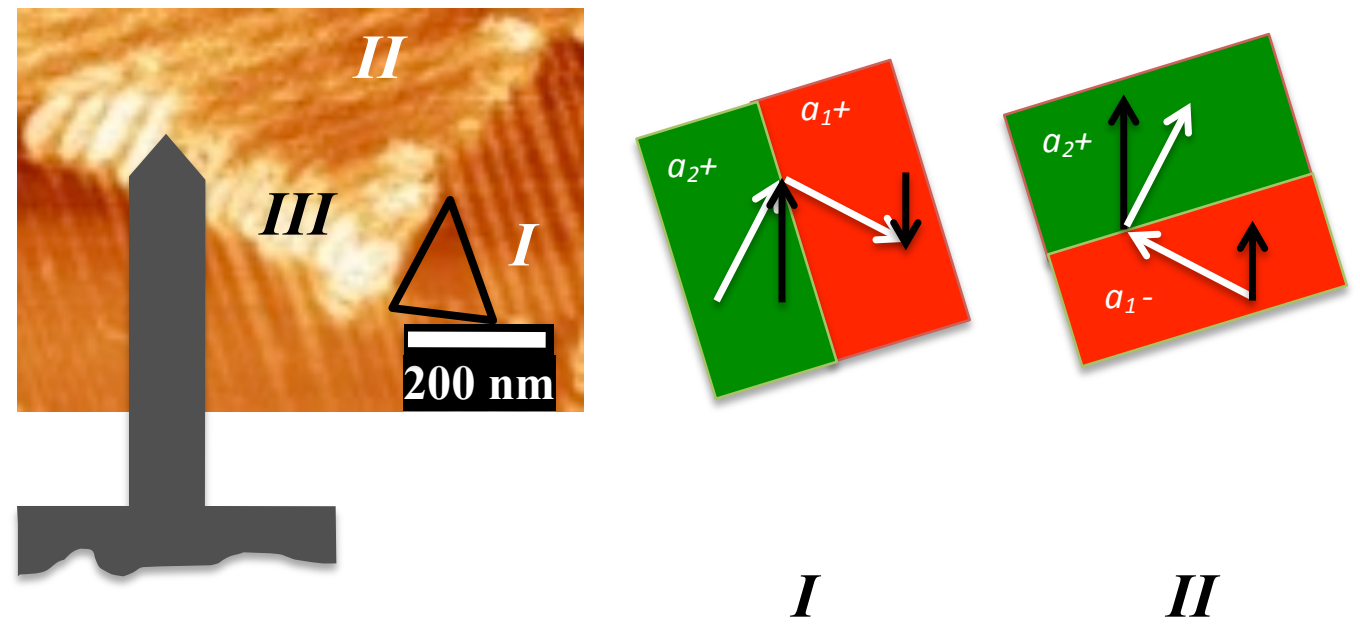

cantilever

Fig. 5 


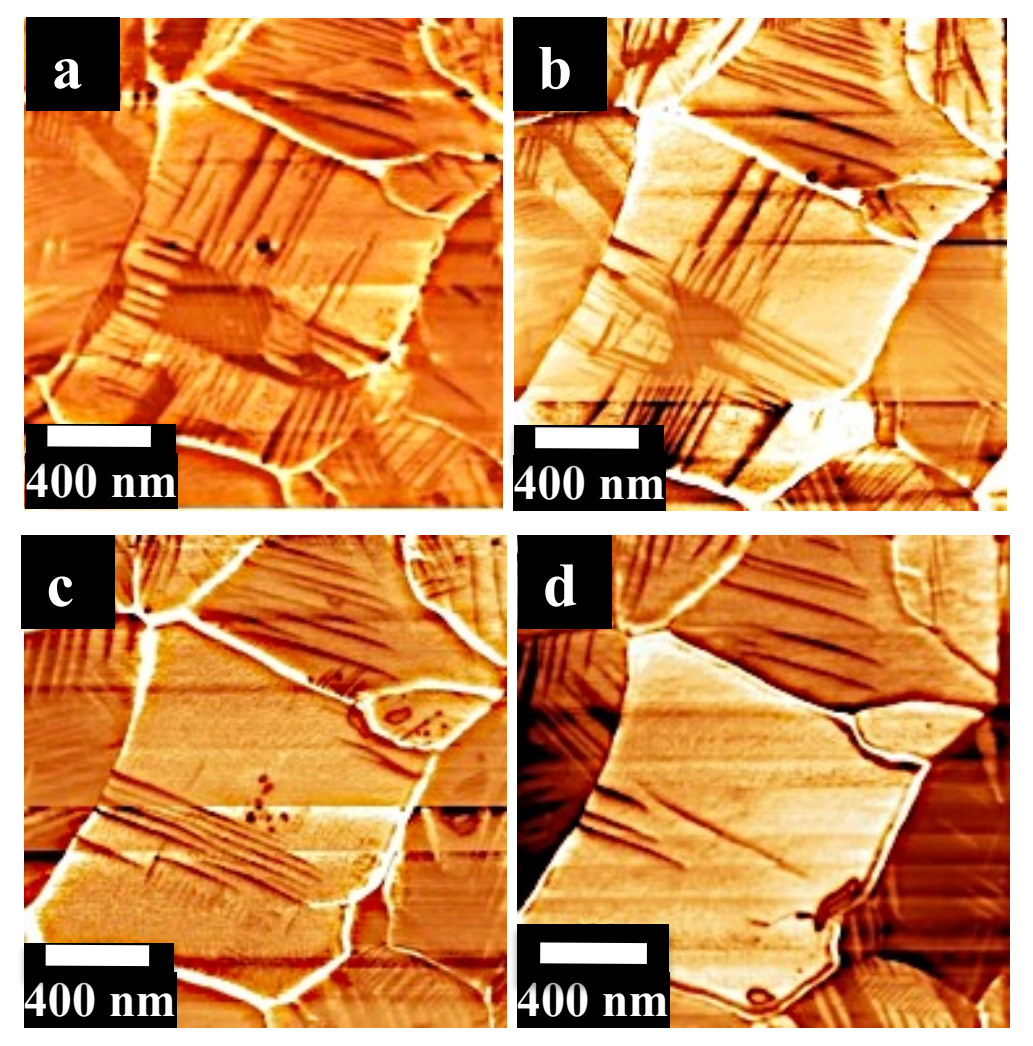

Fig. 6 

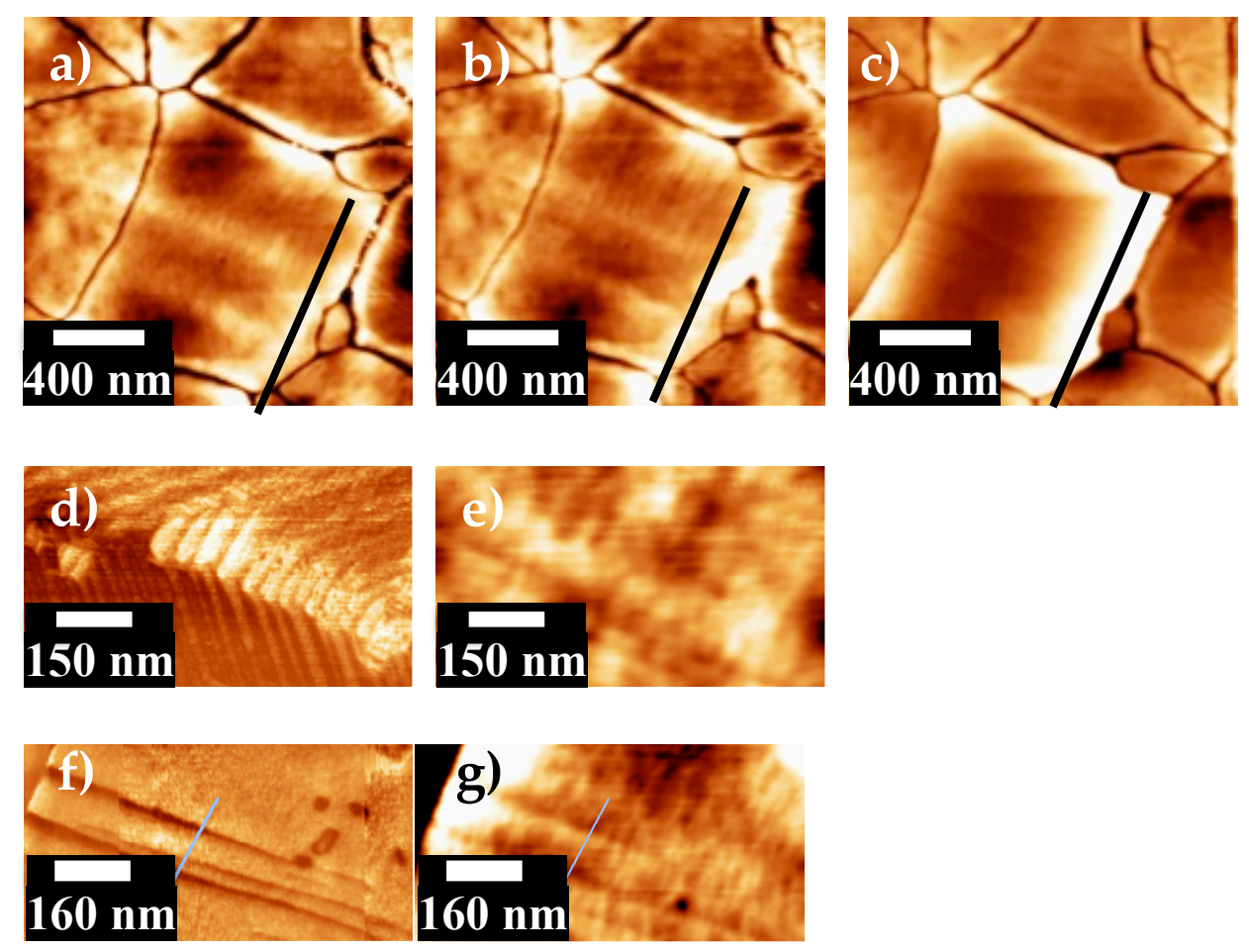

h)

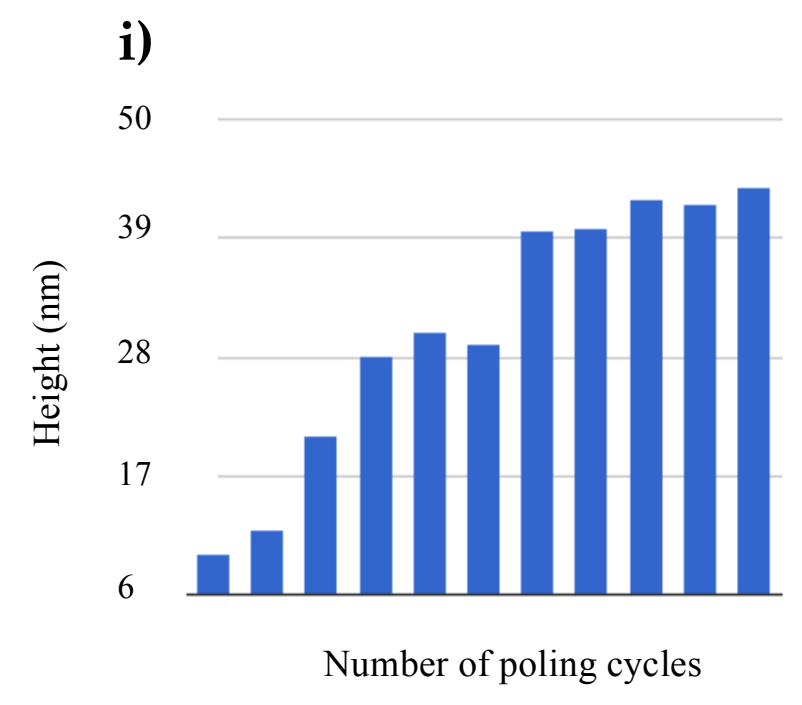

Fig. 7 
a)

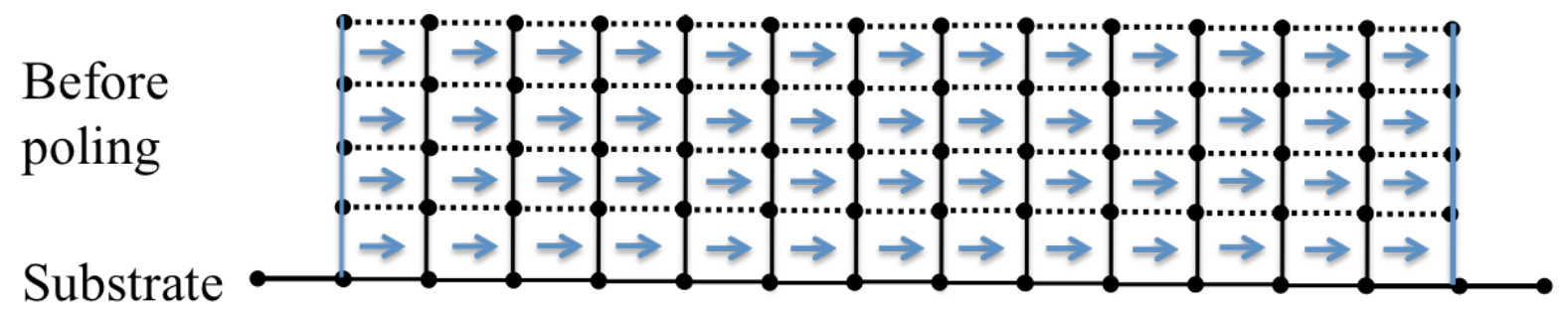

After

poling

Substrate

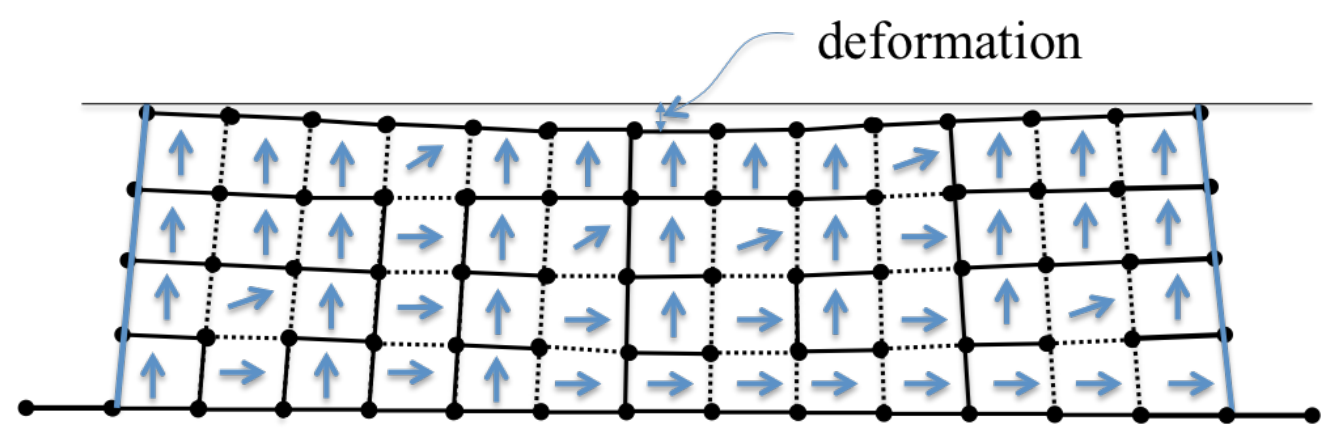

-....... Long axis of unit cell

$\bullet \quad$ Short axis of unit cell

b)

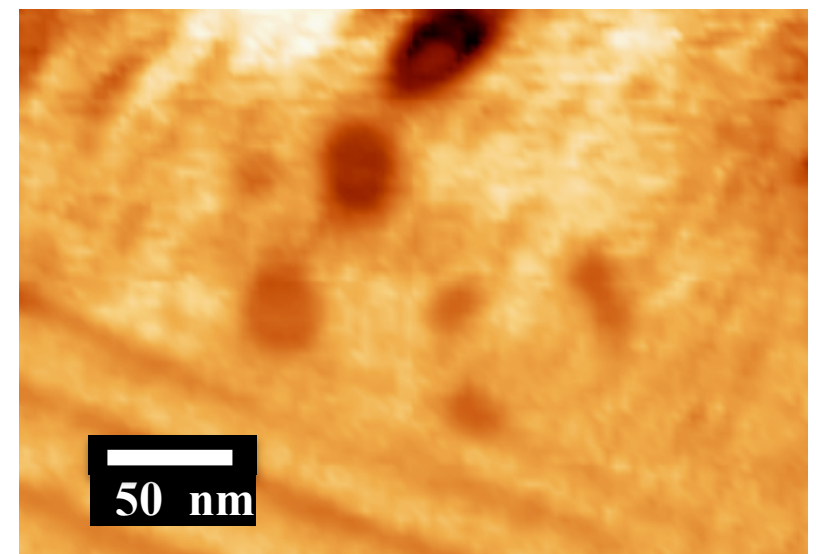

Fig. 8 


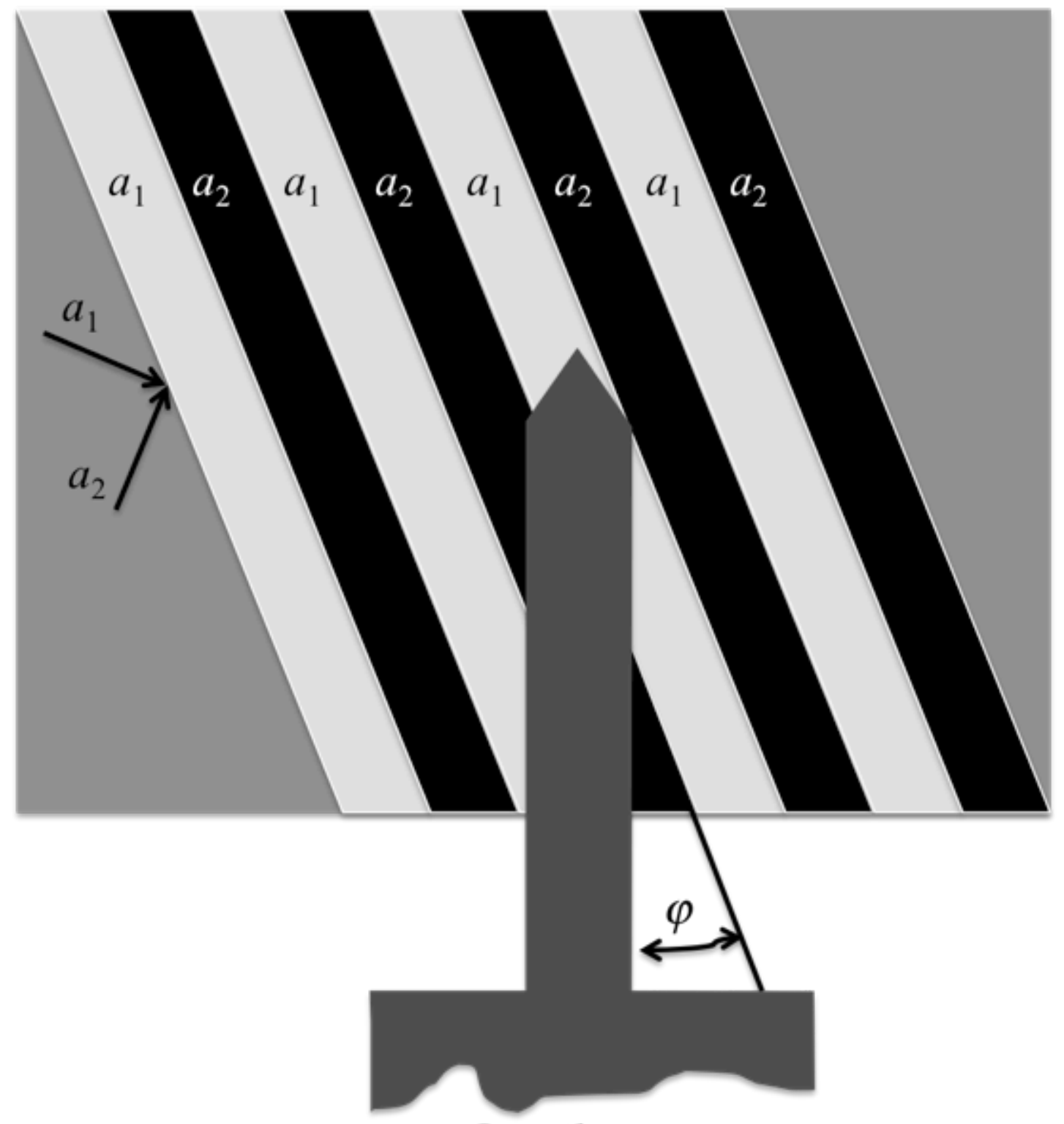

Cantilever

Fig. 9 

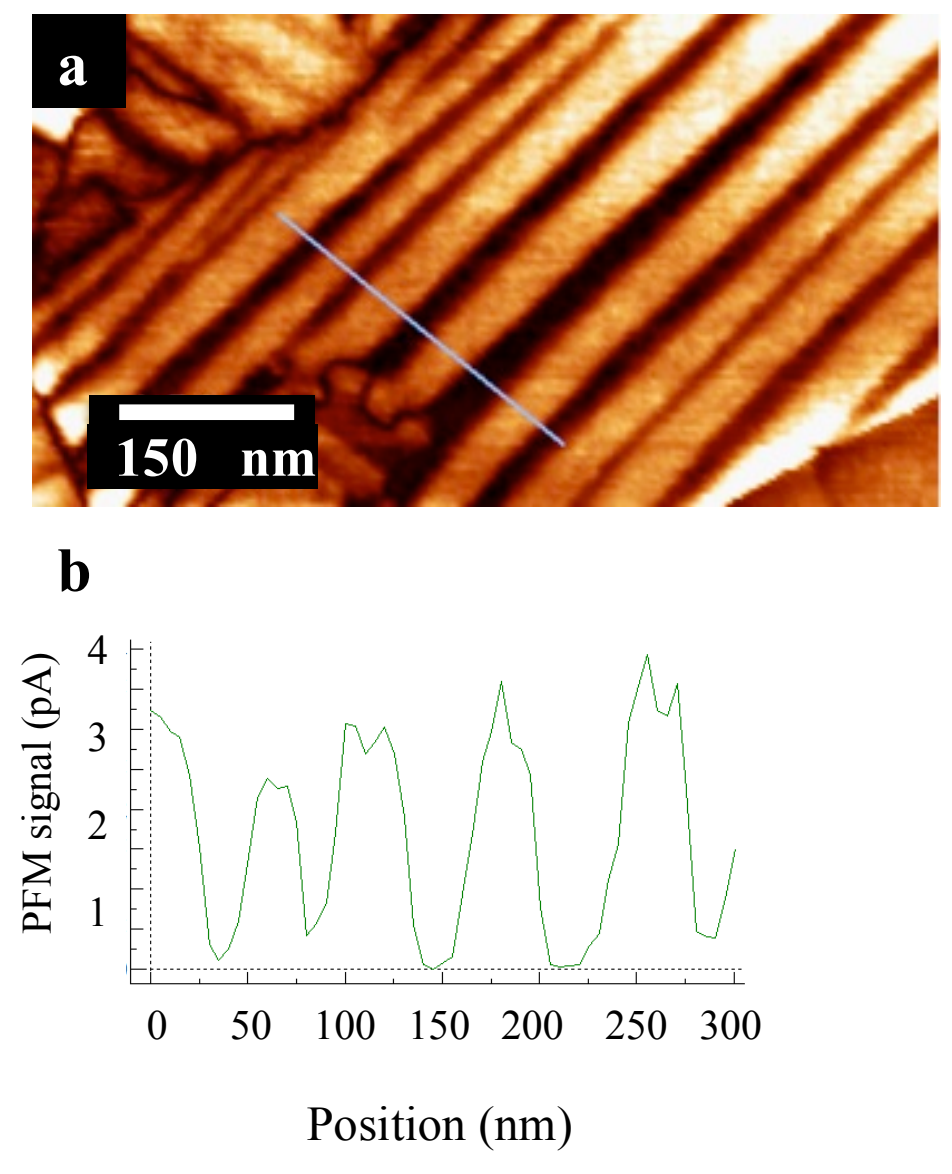

Fig. 10 